\title{
Supplemental Files
}

Group Information

Supplemental Table 1: Associations of Newborn with Childhood Anthropometric Measures by Sex

Supplemental Table 2: Mediation Models for Newborn Fat Mass z-score

Supplemental Table 3: Mediation Models for Birthweight z-score and Birthweight-for-length z-score

HAPO FUS Group Information: The collaborator members of the HAPO Follow-up Study Cooperative Research Group by field center are: Bangkok, Thailand: C. Deerochanawong, T. Tanaphonpoonsuk (Rajavithi Hospital) and S. Binratkaew, U. Chotigeat, W. Manyam (Queen Sirikit National Institute of Child Health); Barbados: M. Forde, A. Greenidge, K. Neblett, P. M. Lashley, D. Walcott (Queen Elizabeth Hospital, School of Clinical Medicine and Research, University of the West Indies); Belfast, Ireland: K. Corry, L. Francis, J. Irwin, A. Langan, D. R. McCance, M. Mousavi (Belfast Health and Social Care Trust) and I. S. Young (Queen's University); Bellflower, California: J. Gutierrez, J. Jimenez, J. M. Lawrence, D. A. Sacks, H. S. Takhar, E. Tanton (Kaiser Permanente of Southern California); Chicago, Illiniois: W. J. Brickman, J. Howard, J. L. Josefson, L. Miller (Ann and Robert H. Lurie Children's Hospital and Northwestern University Feinberg School of Medicine); Cleveland, Ohio: J. Bjaloncik, P. M. Catalano, A. Davis, K. Koontz, L. Presley, S. Smith, A. Tyhulski (MetroHealth Medical Center and Case Western Reserve University); Hong Kong, China: A. Li, R. C. Ma, R. Ozaki, W. H. Tam, M. Wong, C. Yuen (Chinese University of Hong Kong and Prince of Wales Hospital); Manchester, England: P. E. Clayton, A. Khan, A. Vyas (Royal Manchester Children's Hospital, Manchester University Hospitals NHS Foundation Trust, Manchester Academic Healthy Sciences Centre and School of Medical Sciences, Faculty of Biology, Medicine, and Health, University of Manchester) and M. Maresh (St Mary's Hospital, Manchester University Hospitals NHS Foundation Trust, Manchester Academic Health Sciences Centre); Petah-Tikva, Isreal: H. Benzaquen, N. Glickman, A. Hamou, O. Hermon, O. Horesh, Y. Keren, S. Shalitin (Schneider Children's Medical Center of Israel) and Y. Lebenthal (Jesse Z. and Sara Lea Shafer Institute for Endocrinology and Diabetes, National Center for Childhood Diabetes, Schneider Children's Medical Center of Israel, Sackler Faculty of Medicine, Tel Aviv University); and Toronto, Ontario, Canada: K. Cordeiro, J. Hamilton, H. Y. Nguyen, S. Steele (Hospital for Sick Children, University of Toronto). Coordinating Center: Northwestern University Feinberg School of Medicine (F. Chen, A. R. Dyer, W. Huang, A. Kuang, M. Jimenez, L. P. Lowe, W. L. Lowe Jr, B. E. Metzger, M. Nodzenski, A. Reisetter, D. Scholtens, O. Talbot, P. Yim). Consultants: D. Dunger, A. Thomas. National Institute of Diabetes and Digestive and Kidney Diseases: M. Horlick, B. Linder, A. Unalp-Arida. Eunice Kennedy Shriver National Institute of Child Health and Human Development: G. Grave. 
Supplemental Table 1: Associations of Newborn with Childhood Anthropometric Measures by Sex

\begin{tabular}{|c|c|c|}
\hline \multirow[b]{2}{*}{$\begin{array}{l}\text { Childhood adiposity } \\
\text { Outcome }\end{array}$} & \multicolumn{2}{|c|}{ Adjusted Mean Difference $(95 \% \mathrm{Cl}, \mathrm{p})$} \\
\hline & Female & Male \\
\hline & \multicolumn{2}{|c|}{ Newborn sum of skinfolds z-score } \\
\hline $\mathrm{BMI}\left(\mathrm{kg} / \mathrm{m}^{2}\right)$ & $0.19(0.0012-0.38,0.049)$ & $0.34(0.15-0.53,<0.001)$ \\
\hline BMI z-score & $0.052(-0.0023-0.11,0.061)$ & $0.097(0.041-0.15,<0.001)$ \\
\hline Fat mass $(\mathrm{kg})$ & $0.41(0.073-0.76,0.017)$ & $0.64(0.27-1.010,<0.001)$ \\
\hline Percent bodyfat (\%) & $0.55(0.098-0.99,0.017)$ & $0.75(0.25-1.26,0.0037)$ \\
\hline \multirow[t]{2}{*}{ Sum of Skinfolds (mm) } & $0.83(-0.12-1.77,0.086)$ & $1.53(0.46-2.60,0.0051)$ \\
\hline & \multicolumn{2}{|l|}{ Newborn fat mass z-score } \\
\hline $\mathrm{BMI}\left(\mathrm{kg} / \mathrm{m}^{2}\right)$ & $0.24(0.046-0.44,0.016)$ & $0.36(0.17-0.55,<0.001)$ \\
\hline BMI z-score & $0.10(0.045-0.16,<0.001)$ & $0.13(0.073-0.19,<0.001)$ \\
\hline Fat mass $(\mathrm{kg})$ & $0.32(-0.033-0.68,0.076)$ & $0.41(0.040-0.79,0.030)$ \\
\hline Percent bodyfat (\%) & $0.33(-0.14-0.80,0.16)$ & $0.34(-0.17-0.85,0.19)$ \\
\hline \multirow[t]{2}{*}{ Sum of Skinfolds $(\mathrm{mm})$} & $-0.27(-1.30-0.71,0.59)$ & $0.64(-0.42-1.71,0.24)$ \\
\hline & \multicolumn{2}{|l|}{ z-score } \\
\hline BMI $\left(\mathrm{kg} / \mathrm{m}^{2}\right)$ & $0.25(0.028-0.47,0.027)$ & $0.41(0.20-0.61,<0.001)$ \\
\hline BMI z-score & & $0.15(0.088-0.21,<0.001)$ \\
\hline Fat mass $(\mathrm{kg})$ & $0.43(0.034-0.82,0.033)$ & $0.58(0.18-0.97,0.0044)$ \\
\hline Percent bodyfat (\%) & & $0.38(-0.17-0.93,0.17)$ \\
\hline \multirow{2}{*}{ Sum of Skinfolds (mm) } & $-0.35(-1.40-0.73,0.52)$ & $0.67(-0.46-1.80,0.24)$ \\
\hline & \multicolumn{2}{|c|}{ Newborn birthweight-for-length z-score } \\
\hline BMI $\left(\mathrm{kg} / \mathrm{m}^{2}\right)$ & $0.16(0.018-0.31,0.028)$ & $0.13(-0.012-0.27,0.073)$ \\
\hline BMI z-score & $0.070(0.028$ & $0.058(0.017-0.10,0.0056)$ \\
\hline Fat mass $(\mathrm{kg})$ & $0.0024(-0.26-$ & $-0.11(-0.38-0.16,0.42)$ \\
\hline Percent bodyfat (\%) & $-0.094(-0.44-0.25,0.60)$ & $-0.14(-0.51-0.23,0.46)$ \\
\hline \multirow[t]{2}{*}{ Sum of Skinfolds (mm) } & $-0.24(-0.96-0.49,0.52)$ & $-0.00031(-0.76-0.76,0.999)$ \\
\hline & \multicolumn{2}{|l|}{ Newborn log cord c-peptide } \\
\hline BMI $\left(\mathrm{kg} / \mathrm{m}^{2}\right)$ & $0.10(-0.078-0.28,0.27)$ & $0.17(-0.00063-0.35,0.051)$ \\
\hline BMI z-score & $0.014(-0.038-0.065,0.60)$ & $0.056(0.0044-0.11,0.034)$ \\
\hline Fat $n$ & $0.24(-0.073-0.56,0.13)$ & $0.29(-0.045-0.63,0.090)$ \\
\hline Percent bodyfat (\%) & $0.28(-0.15-0.70,0.20)$ & $0.59(0.12-1.050,0.013)$ \\
\hline Sum of Skinfolds $(\mathrm{mm})$ & $0.62(-0.26-1.50,0.17)$ & $1.10(0.13-2.080,0.027)$ \\
\hline
\end{tabular}

All adjusted mean differences in childhood anthropometric measures are reported for each newborn measure higher by 1 standard deviation.

Model 2: field center, child's sex, maternal gestational age at delivery, maternal age, mean arterial pressure, height, parity, smoking, drinking, and family history of diabetes at pregnancy OGTT, maternal glucose sum of z-scores and BMI at pregnancy OGTT 
Supplemental Table 2: Mediation Models for Newborn Fat Mass z-score

\begin{tabular}{|c|c|c|c|c|}
\hline $\begin{array}{l}\text { Maternal } \\
\text { Exposure }\end{array}$ & $\begin{array}{l}\text { Direct Effect: Beta (95\% } \\
\text { Cl, p) }\end{array}$ & $\begin{array}{l}\text { Indirect Effect: Beta } \\
(95 \% \mathrm{Cl}, \mathrm{p})\end{array}$ & $\begin{array}{l}\text { Total Effect: Beta }(95 \% \\
\text { Cl, p) }\end{array}$ & $\begin{array}{l}\text { Proportion } \\
\text { Mediated }\end{array}$ \\
\hline \multicolumn{5}{|c|}{ Childhood BMI $\left(\mathrm{kg} / \mathrm{m}^{2}\right)$} \\
\hline $\begin{array}{l}\text { Glucose Sum of } \\
\text { z-scores }\end{array}$ & $\begin{array}{c}0.024(-0.033-0.081 \\
0.41)\end{array}$ & $\begin{array}{c}0.017(0.0081-0.026, \\
<0.001)\end{array}$ & $\begin{array}{c}0.041(-0.016-0.098 \\
0.16)\end{array}$ & * \\
\hline BMI & $0.30(0.27-0.33,<0.001)$ & 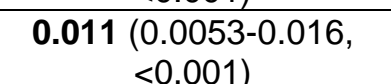 & $0.31(0.28-0.34,<0.001)$ & 0.035 \\
\hline \multicolumn{5}{|c|}{ Childhood BMI z-scores } \\
\hline $\begin{array}{l}\text { Glucose Sum of } \\
\text { z-scores }\end{array}$ & $\begin{array}{c}0.00066(-0.016-0.017 \\
0.94)\end{array}$ & $\begin{array}{c}0.0065(0.0038-0.0092 \\
<0.001)\end{array}$ & $\begin{array}{c}0.0072(-0.0093-0.024 \\
0.39)\end{array}$ & * \\
\hline BMI & $\begin{array}{c}0.083(0.074-0.091 \\
<0.001)\end{array}$ & $\begin{array}{c}0.0041(0.0025-0.0057 \\
<0.001)\end{array}$ & $\begin{array}{c}0.087(0.078-0.095 \\
<0.001)\end{array}$ & 0.047 \\
\hline \multicolumn{5}{|c|}{ Childhood fat mass (kg) } \\
\hline $\begin{array}{l}\text { Glucose Sum of } \\
\text { z-scores }\end{array}$ & $\begin{array}{c}0.14(0.036-0.25 \\
0.0088)\end{array}$ & $\begin{array}{c}\mathbf{0 . 0 2 0}(0.0043-0.035, \\
0.012)\end{array}$ & $0.16(0.057-0.27,0.0027)$ & 0.12 \\
\hline BMI & $0.47(0.42-0.53,<0.001)$ & $\begin{array}{c}\mathbf{0 . 0 1 2}(0.0028-0.022, \\
0.011)\end{array}$ & $0.49(0.43-0.54,<0.001)$ & 0.024 \\
\hline \multicolumn{5}{|c|}{ Childhood percent body fat (\%) } \\
\hline $\begin{array}{l}\text { Glucose Sum of } \\
\text { z-scores }\end{array}$ & $0.28(0.13-0.42,<0.001)$ & $\begin{array}{c}0.017(-0.0035-0.037 \\
0.11)\end{array}$ & $0.29(0.15-0.44,<0.001)$ & $\wedge$ \\
\hline BMI & $0.52(0.45-0.59,<0.001)$ & $\begin{array}{c}0.010(-0.0021-0.023 \\
0.10)\end{array}$ & $0.53(0.46-0.60,<0.001)$ & $\wedge$ \\
\hline \multicolumn{5}{|c|}{ Childhood sum of skinfolds (mm) } \\
\hline $\begin{array}{l}\text { Glucose Sum of } \\
\text { z-scores }\end{array}$ & $0.52(0.21-0.82,<0.001)$ & $\begin{array}{c}0.0080(-0.033-0.049 \\
0.70)\end{array}$ & $0.52(0.22-0.82,<0.001)$ & $\wedge$ \\
\hline BMI & $\begin{array}{c}1.20(1.050-1.36, \\
<0.001)\end{array}$ & $\begin{array}{c}0.0053(-0.021-0.032 \\
0.70) \\
\end{array}$ & $1.21(1.060-1.36,<0.001)$ & $\wedge$ \\
\hline
\end{tabular}

*Total effect not significant

$\wedge$ Indirect effect not significant 
Supplemental Table 3: Mediation Models for Newborn Birthweight Z-score and Birthweight-for-length Z-score

\begin{tabular}{|c|c|c|c|c|c|}
\hline $\begin{array}{l}\text { Maternal } \\
\text { Exposure }\end{array}$ & $\begin{array}{l}\text { Newborn } \\
\text { Outcome }\end{array}$ & $\begin{array}{c}\text { Direct Effect: Beta (95\% } \\
\text { Cl, p value) }\end{array}$ & $\begin{array}{c}\text { Indirect Effect: Beta (95\% } \\
\text { Cl, p value) }\end{array}$ & $\begin{array}{c}\text { Total Effect: Beta }(95 \% \\
\text { Cl, p value) }\end{array}$ & $\begin{array}{c}\text { Proportion } \\
\text { Mediated }\end{array}$ \\
\hline \multicolumn{6}{|c|}{ Outcome: Childhood BMI $\left(\mathrm{kg} / \mathrm{m}^{2}\right)$} \\
\hline \multirow{2}{*}{$\begin{array}{l}\text { Glucose Sum } \\
\text { of z-scores }\end{array}$} & Birthweight & $\begin{array}{c}0.015(-0.039-0.068, \\
0.59)\end{array}$ & $\begin{array}{c}0.018(0.0090-0.027, \\
<0.001)\end{array}$ & $\begin{array}{c}0.033(-0.020-0.086, \\
0.23)\end{array}$ & * \\
\hline & $\begin{array}{l}\text { Birthweight- } \\
\text { for-length }\end{array}$ & $\begin{array}{c}0.030(-0.024-0.084, \\
0.28) \\
\end{array}$ & $\begin{array}{c}0.0041(0.00040-0.0077 \\
0.030)\end{array}$ & $\begin{array}{c}0.034(-0.020-0.088, \\
0.22) \\
\end{array}$ & * \\
\hline \multirow{2}{*}{$\mathrm{BMI}\left(\mathrm{kg} / \mathrm{m}^{2}\right)$} & Birthweight & $0.29(0.27-0.32,<0.001)$ & $\begin{array}{c}0.010(0.0052-0.015 \\
<0.001)\end{array}$ & $0.30(0.28-0.33,<0.001)$ & 0.033 \\
\hline & $\begin{array}{l}\text { Birthweight- } \\
\text { for-length }\end{array}$ & $0.30(0.27-0.33,<0.001)$ & $\begin{array}{c}0.0033(0.00065-0.0059 \\
0.014)\end{array}$ & $0.30(0.28-0.33,<0.001)$ & 0.011 \\
\hline \multicolumn{6}{|c|}{ Childhood BMI z-score } \\
\hline \multirow{2}{*}{$\begin{array}{l}\text { Glucose Sum } \\
\text { of } z \text {-scores }\end{array}$} & Birthweight & $\begin{array}{c}-0.0010(-0.017-0.015 \\
0.90)\end{array}$ & $\begin{array}{c}0.0073(0.0046-0.010, \\
<0.001)\end{array}$ & $\begin{array}{c}0.0063(-0.0091-0.022 \\
0.42)\end{array}$ & * \\
\hline & $\begin{array}{l}\text { Birthweight- } \\
\text { for-length }\end{array}$ & $\begin{array}{c}0.0046(-0.011-0.020 \\
0.56)\end{array}$ & $\begin{array}{c}0.0018(0.00053-0.0031 \\
0.0055)\end{array}$ & $\begin{array}{c}0.0064(-0.0092-0.022 \\
0.42)\end{array}$ & * \\
\hline \multirow{2}{*}{ BMI $\left(\mathrm{kg} / \mathrm{m}^{2}\right)$} & Birthweight & $\begin{array}{c}0.083(0.074-0.091, \\
<0.001)\end{array}$ & $\begin{array}{c}0.0042(0.0025-0.0057 \\
<0.001)\end{array}$ & $\begin{array}{c}0.087(0.078-0.095, \\
<0.001)\end{array}$ & 0.048 \\
\hline & $\begin{array}{l}\text { Birthweight- } \\
\text { for-length }\end{array}$ & $\begin{array}{c}0.085(0.077-0.093, \\
<0.001)\end{array}$ & $\begin{array}{c}0.0015\left(\begin{array}{c}0.00062-0.0023, \\
<0.001)\end{array}\right. \\
\end{array}$ & $\begin{array}{c}0.087 \begin{array}{c}(0.079-0.094, \\
<0.001)\end{array} \\
\end{array}$ & 0.017 \\
\hline \multicolumn{6}{|c|}{ Childhood fat mass $(\mathrm{kg})$} \\
\hline \multirow{2}{*}{$\begin{array}{l}\text { Glucose Sum } \\
\text { of } z \text {-scores }\end{array}$} & Birthweight & $0.13(0.027-0.23,0.013)$ & $\begin{array}{c}0.027(0.010-0.043, \\
0.0013) \\
\end{array}$ & $0.15(0.054-0.25,0.0024)$ & 0.18 \\
\hline & $\begin{array}{l}\text { Birthweight- } \\
\text { for-length }\end{array}$ & $\begin{array}{c}0.16(0.058-0.26 \\
0.0020)\end{array}$ & $\begin{array}{c}-0.0023(-0.0080-0.0034 \\
0.43)\end{array}$ & $0.16(0.056-0.26,0.0023)$ & $\wedge$ \\
\hline \multirow{2}{*}{$\mathrm{BMI}\left(\mathrm{kg} / \mathrm{m}^{2}\right)$} & Birthweight & $.51,<0.001)$ & $\begin{array}{c}0.015(0.0058-0.024 \\
0.0012)\end{array}$ & $0.47(0.42-0.52,<0.001)$ & 0.032 \\
\hline & $\begin{array}{l}\text { Birthweight- } \\
\text { for-length }\end{array}$ & $0.47(0.42-0.52,<0.001)$ & $\begin{array}{c}-0.0018(-0.0063-0.0027 \\
0.43) \\
\end{array}$ & $0.47(0.42-0.52,<0.001)$ & $\wedge$ \\
\hline \multicolumn{6}{|c|}{ Childhood percent bodyfat (\%) } \\
\hline \multirow{2}{*}{$\begin{array}{l}\text { Glucose Sum } \\
\text { of z-scores }\end{array}$} & Birthweight & $0.28(0.14-0.41,<0.001)$ & $\begin{array}{c}0.019(-0.0023-0.040 \\
0.081) \\
\end{array}$ & $0.30(0.16-0.43,<0.001)$ & $\wedge$ \\
\hline & $\begin{array}{l}\text { Birthweight- } \\
\text { for-length }\end{array}$ & $0.30(0.17-0.44,<0.001)$ & $\begin{array}{c}-0.0044(-0.012-0.0035 \\
0.27) \\
\end{array}$ & $0.30(0.16-0.43,<0.001)$ & $\wedge$ \\
\hline \multirow{2}{*}{$\mathrm{BMI}\left(\mathrm{kg} / \mathrm{m}^{2}\right)$} & Birthweight & $0.52(0.45-0.59,<0.001)$ & $\begin{array}{c}0.011(-0.0013-0.022 \\
0.08)\end{array}$ & $0.53(0.46-0.60,<0.001)$ & $\wedge$ \\
\hline & $\begin{array}{l}\text { Birthweight- } \\
\text { for-length }\end{array}$ & $0.53(0.46-0.60,<0.001)$ & $\begin{array}{c}-0.0035(-0.0096-0.0026 \\
0.26) \\
\end{array}$ & $0.53(0.46-0.59,<0.001)$ & $\wedge$ \\
\hline \multicolumn{6}{|c|}{ Childhood sum of skinfolds (mm) } \\
\hline \multirow{2}{*}{$\begin{array}{l}\text { Glucose Sum } \\
\text { of z-scores }\end{array}$} & Birthweight & $0.48(0.20-0.77,<0.001)$ & $\begin{array}{c}0.0067(-0.037-0.051 \\
0.77)\end{array}$ & $0.49(0.21-0.77,<0.001)$ & $\wedge$ \\
\hline & $\begin{array}{l}\text { Birthweight- } \\
\text { for-length }\end{array}$ & $0.48(0.20-0.77,<0.001)$ & $\begin{array}{c}-0.0045(-0.020-0.011 \\
0.55)\end{array}$ & $0.48(0.19-0.76,<0.001)$ & $\wedge$ \\
\hline \multirow{2}{*}{$\mathrm{BMI}\left(\mathrm{kg} / \mathrm{m}^{2}\right)$} & Birthweight & $\begin{array}{c}1.16(1.020-1.30, \\
<0.001)\end{array}$ & $\begin{array}{c}0.0039(-0.022-0.029 \\
0.77)\end{array}$ & $1.16(1.020-1.30,<0.001)$ & $\wedge$ \\
\hline & $\begin{array}{l}\text { Birthweight- } \\
\text { for-length }\end{array}$ & $\begin{array}{c}1.16(1.020-1.31, \\
<0.001)\end{array}$ & $\begin{array}{c}-0.0039(-0.017-0.0088 \\
0.55) \\
\end{array}$ & $1.16(1.020-1.30,<0.001)$ & $\wedge$ \\
\hline
\end{tabular}

${ }^{*}$ Total effect not significant

${ }^{\wedge}$ Indirect effect not significant 